\title{
LOX-1-deficient mice are resistant to zymosan-induced arthritis: A mini review
}

\author{
Kazuhiko Hashimoto ${ }^{*}$, Yutaka Oda ${ }^{1}$, Kotaro Yamagishi ${ }^{1}$, Ichiro Tsukamoto ${ }^{1}$, Masao Akagi ${ }^{1}$ \\ Department of Orthopedic Surgery, Kindai University Hospital, Osaka-Sayama City, Osaka 589-851 1, Japan
}

Article Info

\section{Article Notes}

Received: May 03, 2018

Accepted: June 01, 2018

\section{*Correspondence:}

Dr. Kazuhiko Hashimoto, Department of Orthopedic Surgery, Kindai University Hospital 377-2 Ohno-Higashi, Osaka-

Sayama City, Osaka 589-8511, Japan;

Telephone: +81-072-366-0221;

Fax: +81-072-366-0206;

Email: hazzhiko@med.kindai.ac.jp

(C) 2018 Hashimoto K. This article is distributed under the terms of the Creative Commons Attribution 4.0 International License.

\section{Keywords}

Oxidized low-density lipoprotein (ox-LDL)

Lectin-like

Oxidized low-density lipoprotein receptor-1 (LOX-1)

Zymosan

Arthritis

Osteoarthritis

Atherosclerosis

\section{ABSTRACT}

Background: Some reports have shown that metabolic syndrome, including hypertension, hyperlipidemia, and diabetes mellitus, contributes to osteoarthritis (OA) development. Further, lectin-like oxidized low-density lipoprotein (ox-LDL) and ox-LDL receptor-1 (LOX-1), which contributes to atherosclerosis, have also been considered factors contributing to $\mathrm{OA}$ development. Several studies have suggested that the LOX-1/ox-LDL system is involved in OA development in vitro. We have suggested the same and conducted in vitro and in vivo studies to validate this concept. However, the role of the LOX-1/ox-LDL system in OA development has not been clarified. This study aimed to identify the mechanism of the LOX-1/ox-LDL system to clarify OA development.

Methods: A zymosan-induced arthritis model was used to identify the mechanism of the LOX-1/ox-LDL system using LOX-1-knockout (KO) mice. Zymosan was administered via the intra-articular route to induce arthritis.

Results: From our experiment, we found that the LOX-1/ox-LDL system contributes to OA development through matrix metalloproteinase-3.

Conclusion: Our findings suggest that the treatment of abnormal lipid metabolism may contribute to the prevention and suppression of arthritis.

\section{Introduction}

Osteoarthritis (OA), which is characterized by wear and tear of the articular cartilage, was previously believed to be caused by mechanical stress ${ }^{1}$. However, recent studies have suggested the role of other systemic factors ${ }^{2}$. One of them is metabolic syndrome ${ }^{3 \text {, }}$ 4 , including hypertension, hyperlipidemia, and diabetes mellitus, all of which lead to atherosclerosis ${ }^{5}$. Hyperlipidemia is caused by various factors, such as low levels of high-density lipoprotein (HDL), high levels of low-density lipoprotein (LDL), and triglycerides ${ }^{6}$. In recent years, Sawamura et al. have reported that lectin-like, oxidized low-density lipoprotein (ox-LDL) and ox-LDL receptor-1 (LOX-1) contribute to atherosclerosis ${ }^{7}$. Moreover, recent studies revealed the epidemiological relationship between atherosclerosis and $\mathrm{OA}^{8}$, ${ }^{9}$. However, how atherosclerosis contributes to OA has not been clarified in detail. Interestingly, subsequent studies have shown that the LOX-1/ox-LDL system contributes to the development of rheumatoid arthritis ${ }^{10-12}$. Previously, we described that ox-LDL binding to LOX-1 induces stress-induced premature senescence of chondrocytes and results in suppression of telomerase activity by inactivating the PI3K/Akt pathway ${ }^{13}$. Recently, we also demonstrated that $\mathrm{OA}$ development is downregulated in LOX-1 knockout (KO) 
mice $^{14}$. Moreover, development of age-related OA was also found to be reduced in LOX-1 KO mice ${ }^{15}$.

Generally, arthritis such as rheumatoid arthritis differs from $\mathrm{OA}$, as $\mathrm{OA}$ is induced by mechanical stress ${ }^{16,17}$ while arthritis is mainly induced by inflammation. Although some studies revealed the relationship between arthritis and LOX-1/ox-LDL ${ }^{12,18}$, the roles of LOX-1/ox-LDL system in arthritis are still not clarified in detail. Therefore, this study aimed to clarify the role of the LOX-1/ox-LDL system in the development of arthritis. In other words, we tried to identify the role of LOX-1/ox-LDL in inflammatory changes such as synovitis. We also hypothesized that LOX-1 KO would inhibit arthritis-associated changes, which include synovitis and cartilage degeneration.

\section{Summary of the current study}

\section{Material and methods}

We induced arthritis by administering zymosan intra-articularly. Zymosan is an insoluble fraction of yeast cell walls, which are composed predominantly of polysaccharides. It induces arthritis, which is characterized by cartilage degeneration and synovitis in rodents when injected intraperitoneally or intra-articularly. Zymosan is often used to induce arthritis, especially to induce synovial inflammation ${ }^{19}$. Zymosan was used, as it is the most commonly used method. In addition, in the this study, the model was used to evaluate the role of LOX-1/ox-LDL system on arthritis, especially synovitis.

Wild-type (WT) and LOX-1 KO mice ( $\mathrm{n}=10$, in each) were used, and we injected with zymosan to induce arthritis (180 $\mu \mathrm{g} / 6 \mu \mathrm{L}$; Sigma-Aldrich, St. Louis, MO, USA). We observed at the time point of 24, 48 and 72 hours after zymosan injection. As a control group, we conducted saline injection to contralateral knee joint with observation at the same time point. Immunohistochemical analysis was performed to evaluate the expression of matrix metalloproteinase-1 and 3 (MMP-1 and 3) and to determine its role in arthritis development.

All data have been presented as mean \pm standard deviation. The scores of each group $(n=10)$ were compared using Student's t-test. P-values less than 0.05 were considered significant. All data were analyzed with Stat Mate (Atms, Tokyo, Japan) software for Windows, version $4.01 .15,16)$. The correlation between the LOX-1 or ox-LDL positive cell rate and cartilage degeneration score was examined using Pearson's correlation (Excel 2010, Microsoft, Tokyo, Japan).

\section{Results}

We compared zymosan-induced arthritis in wildtype (WT) and LOX-1 KO mice and found it to be more
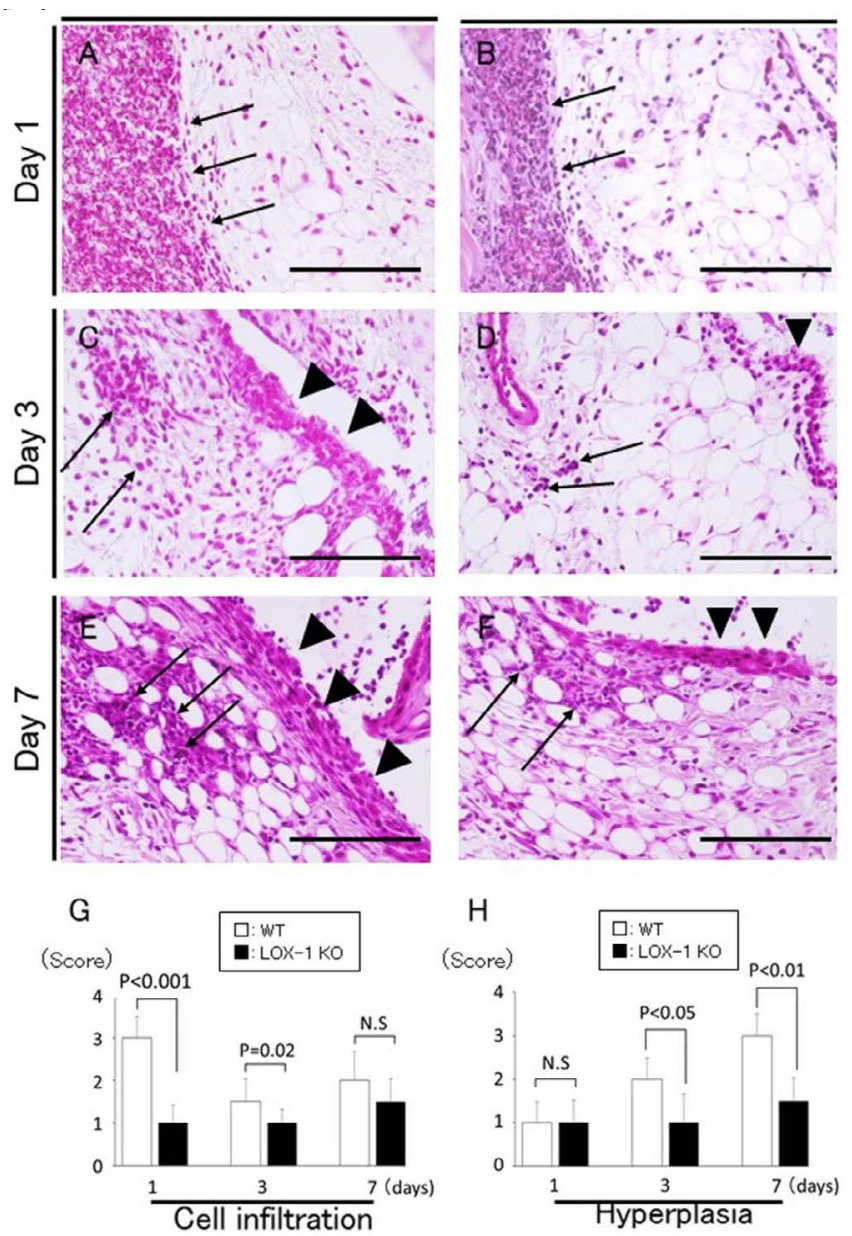

Figure 1: Representative synovium in zymosan-injected group stained with H\&E ( $a-f)$ at $400 \times$ magnification. Synovium of wildtype (WT) mice $(a, c, e)$ and ox-LDL receptor-1 (LOX-1) knockout (KO) mice $(b, d, f)$ at days 1,3 , and 7 after zymosan injection. The graphs show the score of inflammatory cell infiltration $(g)$ and synovial hyperplasia $(h)$ in zymosan-injected groups at each experiment. Inflammatory cell infiltration is observed less in the synovium of the LOX-1 KO mice $(b, d)$ than in that of the WT mice $(a, c)$ at days 1 and 3. Synovial hyperplasia was also observed less in the synovium of the LOX-1 KO mice $(d, f)$ than in that of WT mice $(c, e)$ at days 3 and 7 . Arrows show the infiltrated inflammatory cells in the synovium. Arrowheads show synovial hyperplasia. Data are presented as mean \pm standard deviation $(n=$ 10 , in each group); significant difference $(P<0.05$, Student's t-test) are indicated between the score of the WT and that of LOX-1 KO mice). N.S, not significant. Scale bar $=100 \mu \mathrm{m}$.

severe in the former (Figures 1, 2). Interestingly, both zymosan-induced cartilage degeneration and synovitis were significantly reduced in LOX-1 KO mice compared to WT mice (Figures 1, 2). In the saline-injected control groups, no difference was observed between WT and LOX-1 KO mice (data not shown). Immunohistochemical staining revealed LOX-1 and ox-LDL expression in the chondrocytes and inflammatory synovial cells in WT mice (Figures 3,4 ) but not in LOX-1 KO mice (data not 

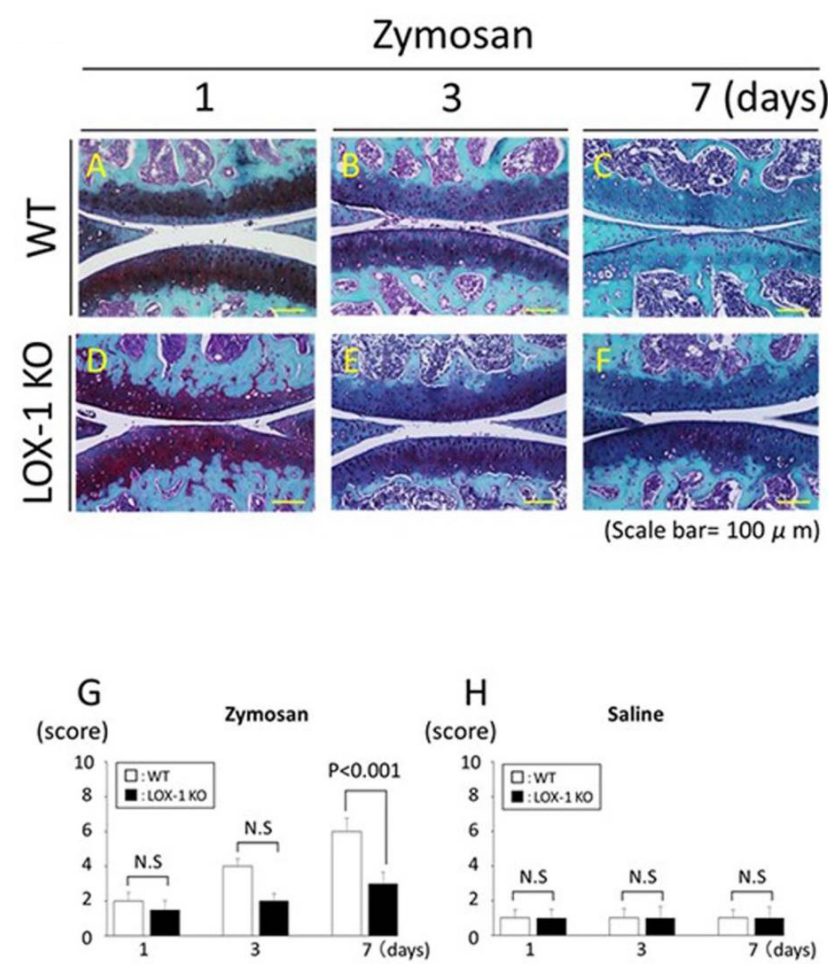

Figure 2: Representative articular cartilage staining with SFO $(a-f)$. Articular cartilage of the wild-type (WT) mice at days 1 , 3 , and 7 after zymosan injection $(a-c)$. Articular cartilage of the ox-LDL receptor-1 (LOX-1) knockout (KO) mice at days 1, 3, and 7 after zymosan injection $(d-f)$. All specimens are in the sagittal direction. The graphs show the score of cartilage degeneration in the zymosan-injected knees $(g)$ and saline-injected knees $(h)$ at each experiment. Cartilage degeneration was less observed in the cartilage of LOX-1 KO mice $(d-f)$ than that in the WT mice $(a-c)$ during all experiments. Data are presented as mean \pm standard deviation ( $n=10$, in each group); $P<0.05$ was regarded as a significant difference (Student's t-test). N.S, not significant. Scale bars $=100 \mu \mathrm{m}$.

shown). These findings suggest that LOX-1/ox-LDL in the chondrocytes and inflammatory synovial cells are involved in the development of arthritis. In our evaluation of the expression of matrix metalloproteinase- 1 and 3 (MMP-1 and 3) by immunohistochemical analysis, MMP-1 was not detected in both WT and LOX-1 KO mice (data not shown); however, MMP-3 expression was significantly higher in the chondrocytes and inflammatory synovial cells of WT mice than in those of LOX-1 KO mice (Figures 5, 6).

\section{Discussion}

The present study was conducted to clarify the role of the LOX-1/ox-LDL system in the development of arthritis, particularly on inflammatory changes such as synovitis. Our findings indicate that MMP-3 plays a role in the development of arthritis downstream of LOX-1/ox-LDL.

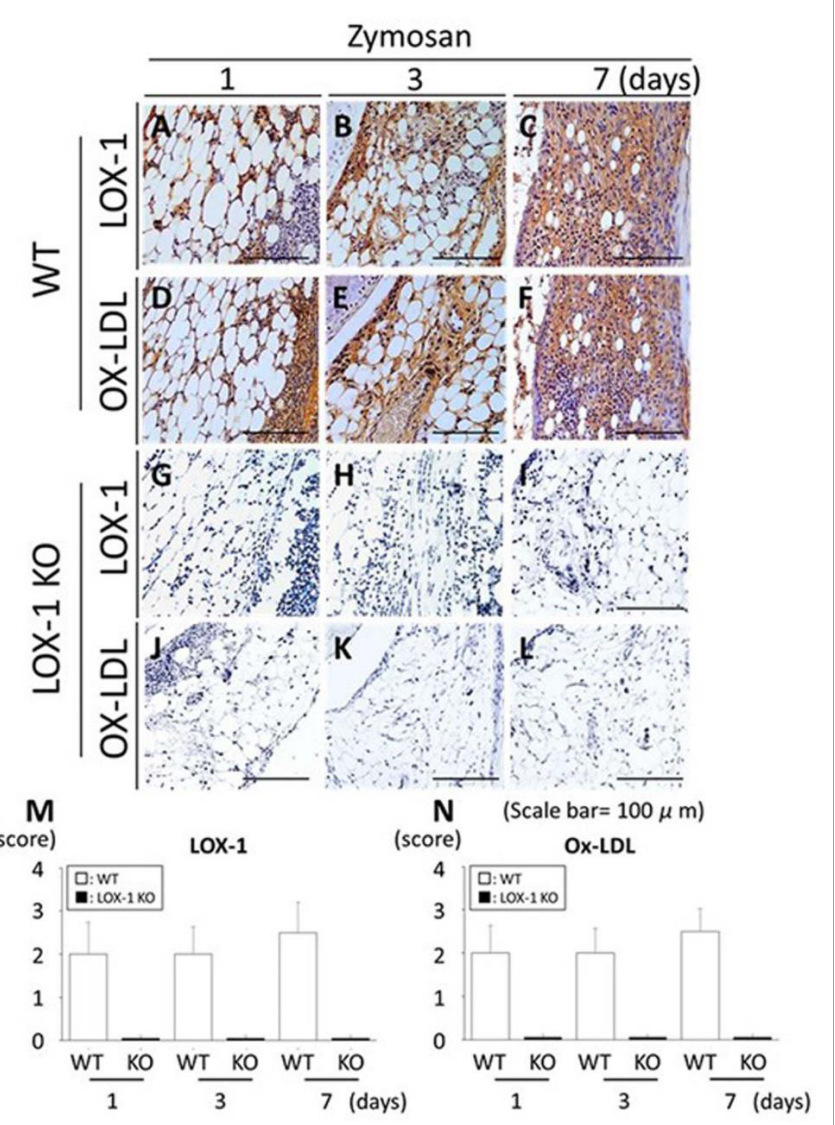

Figure 3: Representative synovial tissues with immunostaining for the ox-LDL receptor-1 (LOX-1) $(a-c)$ and oxidized low-density lipoprotein (ox-LDL) (ox-LDL) (d-f) at 200x magnification. LOX-1 expression in the synovial cells of wild-type (WT) mice at days 1 , 3 and 7 after zymosan injection $(a-c)$. Ox-LDL expression in the synovial cells of WT mice at days 1, 3 and 7 after zymosan injection $(d-f)$. LOX-1 expression in the synovial cells of LOX-1 KO mice at days 1,3 and 7 after zymosan injection $(g-i)$. Ox-LDL expression in the synovial cells of LOX-1 KO mice at days 1,3 and 7 after injection of zymosan $(j-l)$. The graphs show the positive cell score of LOX-1 $(m)$ and ox-LDL $(n)$ expression in the synovium of WT and LOX-1 KO mice at each experiment. Rabbit anti-mouse LOX1 polyclonal and rabbit antimouse ox-LDL polyclonal antibodies were used. Data are presented as mean \pm standard deviation ( $n=$ 10 , in each group). Scale bars $=100 \mu \mathrm{m}$.

These results could provide new findings that LOX-1/oxLDL system is involved in inflammation such as synovitis and cartilage degeneration induced by synovitis.

This study has limitations. First, in vitro methods were not employed, which need to be included in future studies. Second, we could not evaluate systemic inflammation. Zymosan could induce systemic inflammation as previously described $^{20,21}$. In the current study, systemic inflammation as well as the systemic effects of atherosclerosis was not investigated. Second, atherosclerosis was not evaluated. It 

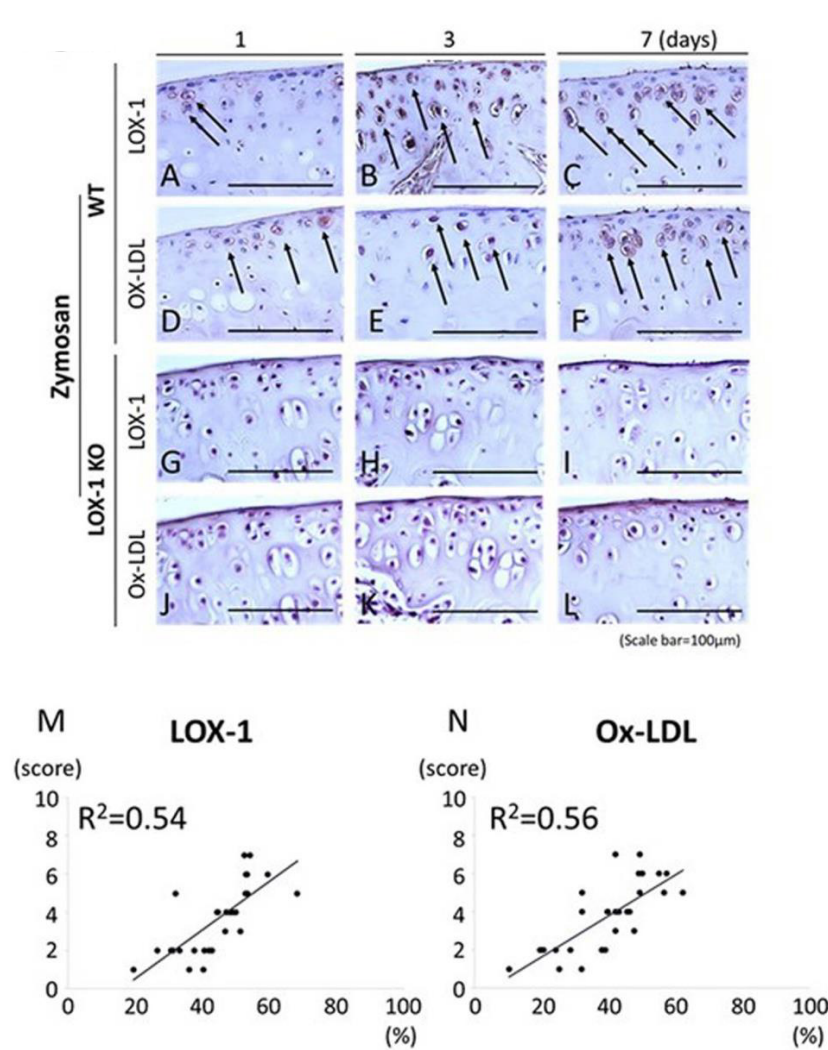

Figure 4: Representative tibial cartilage immunostaining of oxLDL receptor-1 (LOX-1) and oxidized low-density lipoprotein (oxLDL) $(a-l)$ at $400 \times$ magnification. LOX-1 expression in the cartilage of WT mice at days 1,3 , and 7 after zymosan injection $(a-c)$. OxLDL expression in the cartilage of WT mice at days 1,3 and 7 after zymosan injection $(d-f)$. LOX-1 expression in the cartilage of LOX-1 $\mathrm{KO}$ mice at days 1,3 and 7 after zymosan injection $(g-i)$. Ox-LDL expression in the cartilage of LOX-1 KO mice at days 1, 3 and 7 after zymosan injection ( $j-I)$. Although LOX-1 and ox-LDL positive cells are observed in WT mice $(a-f)$, no positive cells are observed in LOX-1 KO mice $(g-l)$ during in all experiments. The graphs show the correlation between the positive cell rate for LOX-1 $(m)$, ox$\operatorname{LDL}(n)$ and the cartilage degeneration score in WT mice. The positive correlation is observed between positive cell rate for LOX-1 or ox-LDL in chondrocytes and the cartilage degeneration score. Arrows show the LOX-1- or ox-LDL-positive chondrocytes. Rabbit anti-mouse LOX-1 polyclonal and rabbit anti-mouse ox-LDL polyclonal antibodies were used. Scale bars $=100 \mu \mathrm{m}$.

would be interesting to investigate the correlation between atherosclerosis and arthritis development. Further research will be necessary.

\section{Conclusion}

Our findings indicate that treatment of abnormal lipid metabolism may contribute to the prevention and suppression of arthritis. Hence, we believe that the LOX-1/ ox-LDL system is involved in the development of arthritis via MMP-3.

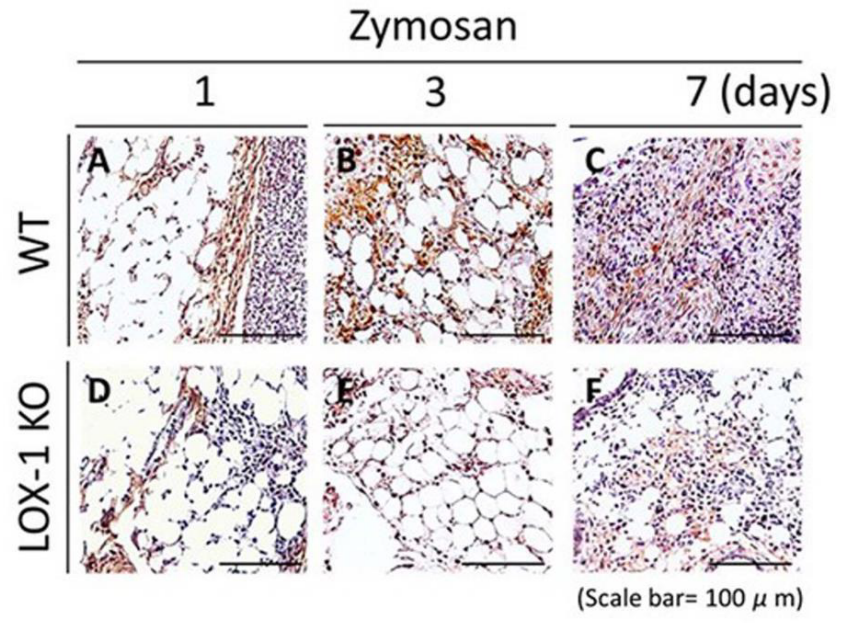

G

(Score)

MMP-3 positive score

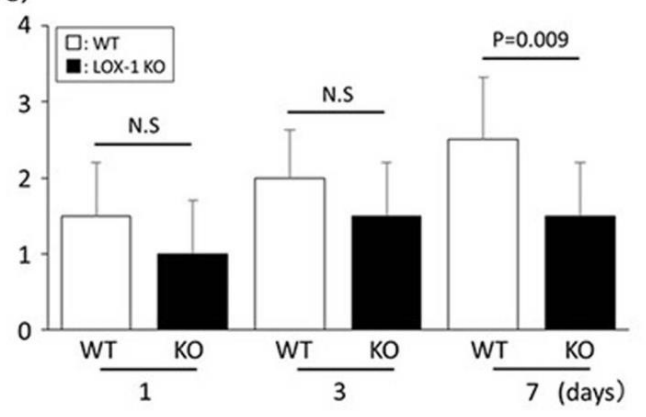

Figure 5: Representative synovial tissues with immunostaining of matrix metalloproteinase-3 (MMP-3) (a-f). MMP-3 expression in the synovial cells of wild-type (WT) mice at days 1, 3 and 7 after zymosan injection $(a-c)$ at $200 \times$ magnification. MMP-3 expression in the synovial cells of ox-LDL receptor-1 (LOX-1) knockout (KO) mice at days 1,3 and 7 after zymosan injection $(d-f)$. MMP3 positive cells are observed both in synovial cells of WT $(a-c)$ and LOX-1 KO mice $(d-f)$ in all experiments. At day 7, MMP-3 in the synovium of LOX-1 KO mice $(f)$ is stained weaker than that in WT mice $(c)$. The graphs show the positive cell score of MMP3 expression in the synovium of WT and LOX-1 KO mice after zymosan injection at each experiment $(g)$. Rabbit anti-mouse MMP-3 polyclonal antibodies were used. Data are presented as mean \pm standard deviation ( $n=10$, in each group). $P<0.05$ was regarded as a significant difference (Student's t-test). N.S, not significant. Scale bars $=100 \mu \mathrm{m}$.

\section{Declarations}

\section{Availability of data and material}

The datasets used and/or analyzed during the current study are available from the corresponding author on reasonable request.

\section{Competing interests}

None. 

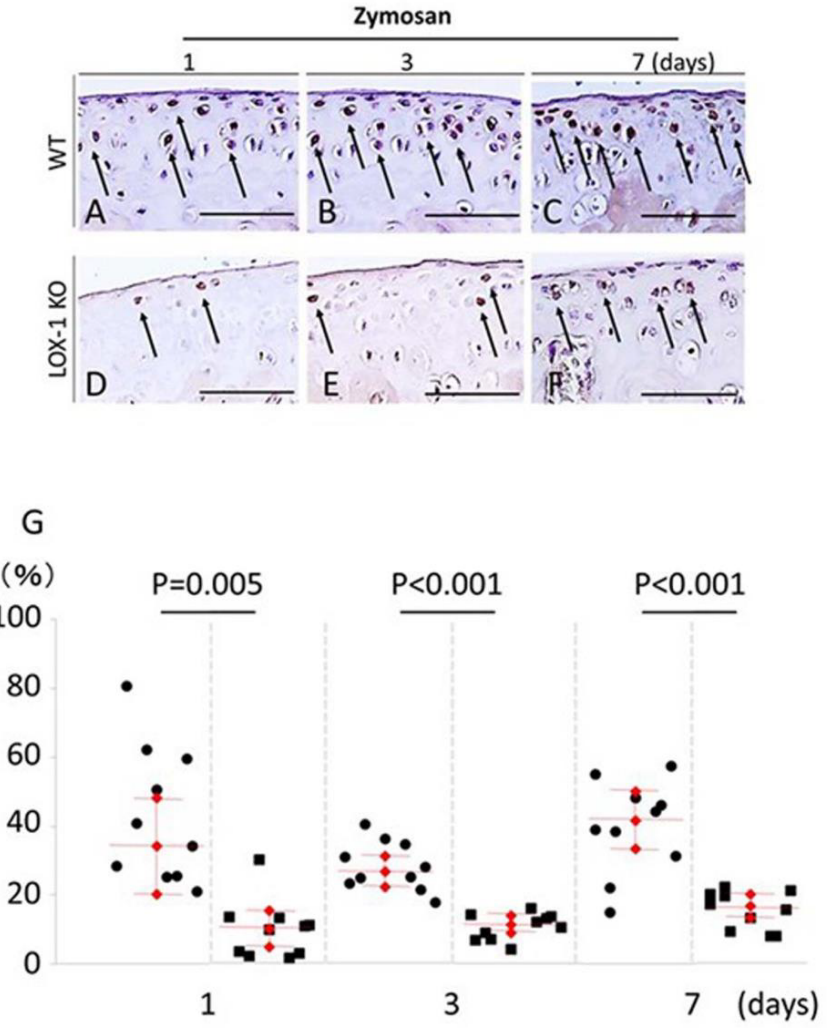

Figure 6: Representative tibial chondrocyte immunostaining of matrix metalloproteinase-3 (MMP-3) (a-f). MMP-3 expression in the chondrocyte of wild-type (WT) mice at days 1,3 , and 7 after zymosan injection $(a-c)$ at $400 \times$ magnification. MMP-3 expression in the chondrocyte of ox-LDL receptor-1 (LOX-1) knockout (KO) mice at days 1,3 and 7 after zymosan injection ( $d-f)$. MMP-3 positive cells are observed in chondrocytes of both WT $(a-c)$ and LOX-1 KO mice $(d-f)$ in all experiments. MMP-3 in chondrocytes of LOX-1 KO mice ( $d-f)$ is stained weaker than that in WT mice ( $a$ $c)$ in all experiments. The graphs show the positive cell score of MMP-3 expression in the chondrocytes of WT and LOX-1 KO mice after zymosan injection at each experiment $(g)$. Arrows show the MMP-3-positive chondrocytes. Rabbit anti-mouse MMP-3 polyclonal antibodies were used. Scale bars $=100 \mathrm{~m}$.

\section{Funding}

This research received no specific grant from any funding agency in the public, commercial, or not-for-profit sectors.

\section{Acknowledgements}

We would like to thank Editage (www.editage.jp) for English language editing.

\section{References}

1. Gofton JP. Studies in osteoarthritis of the hip. 3. Congenital subluxation and osteoarthritis of the hip. Can Med Assoc J. 1971 May 22; 104(10): 911-5.

2. Yoshimura N, Muraki S, Oka H, et al. Mutual associations among musculoskeletal diseases and metabolic syndrome components: A 3-year follow-up of the ROAD study. Mod Rheumatol. 2015 May; 25(3): 438-48.
3. Sun AR, Panchal SK, Friis T, et al. Obesity-associated metabolic syndrome spontaneously induces infiltration of pro-inflammatory macrophage in synovium and promotes osteoarthritis. PloS One. 2017 Aug 31; 12(8): e0183693.

4. Niu J, Clancy M, Aliabadi P, et al. Metabolic syndrome, its components, and knee osteoarthritis: The Framingham Osteoarthritis Study. Arthritis Rheumatol. 2017 Jun; 69(6): 1194-203.

5. Roever L, Resende ES, Diniz ALD, et al. Brazilian Network of Research in Meta-analysis (BRAMETIS). Epicardial adipose tissue and metabolic syndrome: An update protocol for systematic review and meta-analysis. Medicine. 2018 Apr; 97(16): e0387.

6. Nishikido T, Ray KK. Inclisiran for the treatment of dyslipidemia. Expert Opin Investig Drugs. 2018 Mar; 27(3): 287-94.

7. Sawamura T, Kume N, Aoyama T, et al. An endothelial receptor for oxidized low-density lipoprotein. Nature. 1997 Mar 6; 386(6620): 73-7.

8. Hoeven TA, Kavousi M, Ikram MA. Markers of atherosclerosis in relation to presence and progression of knee osteoarthritis: a population-based cohort study.

9. Rheumatology (Oxford, England). 2015 Sep; 54(9):1692-8.

10. Yeh HJ, Chou YJ, Yang NP. Association between physical therapy and risk of coronary artery disease and dyslipidemia among osteoarthritis patients: A nationwide database study. Arch Phys Med Rehab. 2016 Jan; 97(1): 8-16.

11. Akagi M, Nishimura S, Yoshida K, et al. Cyclic tensile stretch load and oxidized low density lipoprotein synergistically induce lectin-like oxidized LDL receptor-1 in cultured bovine chondrocytes, resulting in decreased cell viability and proteoglycan synthesis. J Orthop Res. 2006 Aug; 24(8): 1782-90.

12. Zushi S, Akagi M, Kishimoto H, et al. Induction of bovine articular chondrocyte senescence with oxidized low-density lipoprotein through lectin-like oxidized low-density lipoprotein receptor 1 . Arthritis Rheum. 2009 Oct; 60(10): 3007-16. doi: 10.1002/art.24816.

13. Ishikawa $M$, Ito $H$, Akiyoshi $M$, et al. Lectin-like oxidized low-density lipoprotein receptor 1 signal is a potent biomarker and therapeutic target for human rheumatoid arthritis. Arthritis Rheum. 2012 Apr; 64(4): 1024-34.

14. Kishimoto H, Akagi M, Zushi S, et al. Induction of hypertrophic chondrocyte-like phenotypes by oxidized LDL in cultured bovine articular chondrocytes through increase in oxidative stress. Osteoarthritis Cartilage. 2010 Oct; 18(10): 1284-90.

15. Hashimoto K, Mori S, Oda Y, et al. Lectin-like oxidized low density lipoprotein receptor 1-deficient mice show resistance to instabilityinduced osteoarthritis. Scand J Rheumatol. 2016 Oct; 45(5): 412-22.

16. Hashimoto K, Oda Y, Nakamura F, et al. Lectin-like, oxidized lowdensity lipoprotein receptor-1-deficient mice show resistance to age-related knee osteoarthritis. Eur J Histochem. 2017 Feb 14; 61(1): 2762.

17. Geenen R, Overman CL, Christensen R, et al. EULAR recommendations for the health professional's approach to pain management in inflammatory arthritis and osteoarthritis. Ann Rheum Dis. 2018 Jun; 77(6): 797-807.

18. Lorenz J, Grässel S. Experimental osteoarthritis models in mice. Methods Mol Biol. 2014; 1194: 401-19.

19. Kakinuma T, Yasuda T, Nakagawa T, et al. Lectin-like oxidized lowdensity lipoprotein receptor 1 mediates matrix metalloproteinase 3 synthesis enhanced by oxidized low-density lipoprotein in rheumatoid arthritis cartilage. Arthritis Rheum. 2004 Nov;50(11):3495-3503.

20. Nakagawa T, Akagi M, Hoshikawa H, et al. Lectin-like oxidized low- 
density lipoprotein receptor 1 mediates leukocyte infiltration and articular cartilage destruction in rat zymosan-induced arthritis. Arthritis Rheum. 2002 Sep; 46(9): 2486-2494.
21. Zhang XT, Ding Y, Kang P, et al. Forsythoside A modulates zymosaninduced peritonitis in mice. Molecules. 2018 Mar 6; 23(3): pii: E593. 